\title{
INDEX TO VOLUME 82
}

\section{RESEARCH ANNOUNCEMENTS}

Abikoff, William. Augmented Teichmüller spaces, 333

Ahmad, Shair and Lazer, A. C. Component properties of second order linear systems, 287

Allday, Christopher. The rational homotopy of fixed point sets of torus actions, 893

Altman, Allen B. and Kleiman, Steven L. Compactifying the Jacobian, 947

Anderson, Robert M. A nonstandard representation for Brownian motion and Itô integration, 99

Andreotti, A., Hill, C. D., Kojasiewicz, S. and MacKichan, B. Mayer-Vietoris sequences for complexes of differential operators, 487

Appel, K. and Haken, W. Every planar map is four colorable, 711

Arveson, William. A spectral theorem for nonlinear operators, 511

Aschbacher, Michael. Thin finite simple groups, 484

Aupetit, Bernard. On scarcity of operators with finite spectrum, 485

Bass, H., Connell, E. H. and Wright, D. L. Locally polynomial algebras are symmetric, 719

Baumslag, Gilbert. On the subalgebras of certain finitely presented algebras, 95

Baxendale, Peter. Markov processes on manifolds of maps, 505

Bedford, Eric and Taylor, B. A. The Dirichlet problem for a complex Monge-Ampere equation, 102

Bednarek, A. R. and Ulam, S. M. Generators for algebras of relations, 781

Bell, Harold. A fixed point theorem for plane homeomorphisms, 778

Bellow, Alexandra. Stability properties of the class of asymptotic martingales, 338

Bieri, Robert and Eckmann, Beno. Cobordism for Poincaré duality groups, 137

Birman, Joan S. and Craggs, R. On the $\mu$-invariant of Z-homology 3-spheres, 253

Bloom, Frederick. Stability and growth estimates for Volterra integrodifferential equations in Hilbert space, 603

Brézis, H. and Browder, F. E. Singular Hammerstein equations and maximal monotone operators, 623

- Nonlinear ergodic theorems, 959

Browder, F. E. See Brézis, H.

Caffarelli, Luis A. The regularity of elliptic and parabolic free boundaries, 616

Cantwell, John and Conlon, Lawrence. Open leaves in closed 3-manifolds, 256

Cappell, Sylvain E. and Shaneson, Julius L. Construction of some new four-dimensional manifolds, 69

Casazza, P. G., Kottman, C. A. and Lin, Bor-Luh, On primary Banach spaces, 71

Cavaretta, Alfred S., Jr. One-sided inequalities for the successive derivatives of a function, 303

Cesari, Lamberto. Existence theorems across a point of resonance, 903

Cesari, L. and Suryanarayana, M. B. Existence theorems for Pareto optimization in Banach spaces, 306

Chu, Le Quang. Duality and Kato's theorem on small perturbations, 896

Cline, R. E. and Funderlic, R. E. $A$ theorem on the rank of a difference of matrices, 48

Conlon, Lawrence. See Cantwell, John

Connell, E. H. See Bass, H.

Conway, John B. and Olin, Robert F. A functional calculus for subnormal operators, 259

Craggs, R. See Birman, Joan S.

Damon, James. A partial topological classification for stable map germs, 105

- Topological stability in the nice dimensions $(n \leqslant p), 262$

Dani, S. G. Dynamical systems on homogeneous spaces, 950

Deignan, Daniel. A Wiener-like condition for quasilinear parabolic equations, 309 
Diederich, Klas and Fornaess, John Erik. A strange bounded smooth domain of holomorphy, 74

Diestel, J. and Seifert, C. J. An averaging property of the range of a vector measure, 907

Drake, David A. and Lenz, Hanfried. Finite Hjelmslev planes with new integer invariants, 265

Dvoretzky, Aryeh. On stopping time directed convergence, 347

Eckmann, Beno. See Bieri, Robert.

Edgar, G. A. and Sucheston, L. The Riesz decomposition for vector-valued amarts, 632.

Edwards, David A. and Hastings, Harold M. Whitehead theorems in proper homotopy theory, 59

Generalized Steenrod homology theories, 328

Elliott, Robert J. A stochastic minimum principle, 944

Ferry, Steve. The homeomorphism group of a compact Q-manifold is an ANR, 910

Figiel, T., Lindenstrauss, J. and Milman, V. D. The dimension of almost spherical sections of convex bodies, 575

Fleissner, W. G. See Wage, M. L.

Fornaess, John Erik. See Diederich, Klas

Fraenkel, A. S. and Yesha, Y. Theory of annihilation games, 775

Friedland, S. and Schiffer, M. Global results in control theory with applications to univalent functions, 913

Funderlic, R. E. See Cline, R. E.

Galewski, David E. and Stern, Ronald J. Classification of simplicial triangulations of topological manifolds, 916

Gandulfo, A., Garcia-Cuerva, J. and Taibleson, M. Conjugate system characterizations of $H^{1}$ : counter examples for the Euclidean plane and local fields, 83

Gandulfo, R. O. Transference results for multiplier operators, 734

Garcia-Cuerva, J. See Gandulfo A.

Garg, K. M. On a new definition of derivative, 768

Gariepy, Ronald and Ziemer, William P. A gradient estimate at the boundary for solutions of quasilinear elliptic equations, 629

Garrett, John W. and Stanojević, Časlav V. On $L^{1}$ convergence of certain cosine sums, 129

- Integrability of shifted trigonometric series, 252

Gentry, R. D. and Travis, C. C. Existence and comparison of eigenvalues of nth order linear differential equations, 350

Gérardin, Paul. Three Weil representations associate to finite fields, 268

Glimm, J., Jaffe, A. and Spencer, T. Phase transitions in $P(\phi)_{2}$ quantum fields, 713

Gluck, Herman. See Singer, David

Gluck, Herman and Singer, David. Deformations of geodesic fields, 571

Gohberg, I., Kaashoek, M. A. and Lay, D. C. Spectral classification of operators and operator functions, 587

Gohberg, I. C. and Lerer, L. E. Resultants of matrix polynomials, 565

Goodearl, K. R. Direct sum properties of quasi-injective modules, 108

Gordon, Robert and Green, Edward L. Indecomposable modules: modules with cores, 590

- Indecomposable modules: amalgamations, 884

Green, Edward L. See Gordon, Robert

Greenlee, W. M. Singular perturbation theory for semibounded operators, 341

Grove, Karsten and Tanaka, Minoru. On the number of invariant closed geodesics, 497

Grübaum, F. Alberto. Unique factorization in random variables, 53

Guccione, S. J. and Stanojević, C. V. Probabilistic foundations of quantum theories and Rubin-Stone spaces, 481

Guillemin, Victor and Weinstein, Alan. Eigenvalues associated with a closed geodesic, 92

Haken, W. See Appel, K.

Hales, Alfred W. Linear analogues of ultrafilters, 311

- Ultrafilters and abelian groups, 554

Harper, John R. and Lin, James P. Two torsion in H-spaces, 612

Harrington, Leo A. and Kechris, Alexander S. On monotone vs. nonmonotone induction, 888

Hastings, Harold M. See Edwards, David A. 
Helffer, Bernard and Rodino, Luigi. Elliptic pseudo differential operators degenerate on a symplectic submanifold, 619

Hill, C. D. See Andreotti, A.

Ho, C. Y. Quadratic pairs for odd primes, 941

Hubbard, John H. and Masur, Howard. On the existence and uniqueness of Strebel differentials, 77

Huff, Melvyn. Conformal maps on Hilbert space, 147

Jaco, William and Shalen, Peter B. Seifert fibered spaces in irreducible sufficiently-large 3manifolds, 765

Jaffe, A. See Glimm, J.

Jech, Thomas and Prikry, Karel. On ideals of sets and the power set operation, 593

Johnson, Russell A. Disintegration of measures on compact transformation groups, 919

$\mathrm{J} \phi$ rgensen, Palle T. Perturbation and analytic continuation of group representations, 921

Kaashoek, M. A. See Gohberg, I.

Kannan, R. and Schuur, J. Boundary value problems for even order nonlinear ordinary differential equations, 80

Kechris, Alexander S. See Harrington, Leo A.

Kleiman, Steven L. See Altman, Allen B.

Klein, Abel. A characterization of Osterwalder-Schrader path spaces by the associated semigroup, 762

Knapp, A. W. Commutativity of intertwining operators. II, 271

Kosniowski, Czes. Generators of the unitary $\mathrm{Z} / p$ bordism ring, 344

Kottman, C. A. See Casazza, P. G.

Krantz, S. G. Optimal Lipschitz and $L^{p}$ estimates for the equation $\bar{\partial} u=f$ on strongly pseudoconvex domains, 51

Kreck, M. Bordism of diffeomorphisms, 759

Lacomba, Ernesto A. Homogeneous mechanical systems with symmetry, and geometry of the coadjoint action, 150

Laderman, Julian D. A noncommutative algorithm for multiplying $3 \times 3$ matrices using 23 multiplications, 126

Lai, K. F. On the Tamagawa number of quasi-split groups, 300

Lance, E. Christopher. A strong noncommutative ergodic theorem, 925

Lay, D. C. See Gohberg, I.

Lazer, A. C. See Ahmad, Shair

de Leeuw, Karel. The failure of spectral analysis in $L^{p}$ for $0<p<1,111$

Lefton, Phyllis. On the Galois groups of cubics and trinomials, 754

Lenz, Hanfried. See Drake, David A.

Lerer, L. E. See Gohberg, I. C.

Levine, L. E. and Obi, W. C. Multivariable expansion of solutions of linear equations with slowly varying coefficients, 771

Lieb, Elliott. Bounds on the eigenvalues of the Laplace and Schroedinger operators, 751

Lin, Bor-Luh. See Casazza, P. G.

Lin, James P. On the cohomology of H-spaces of exceptional type, 277

- See Harper, John R.

Lindenstrauss, J. See Figiel, T.

Loday, Jean-Louis, Higher Whitehead groups and stable homotopy, 134

Lojasiewicz, S. See Andreotti, A.

Lorentz, G. G. Independent knots in Birkhoff interpolation, 783

Lovász, László and Marx, Morris L. A forbidden substructure characterization of Gauss codes, 121

Lucas, W. F. Disconnected solutions, 596

MacBain, John. Global bifurcation theorems for nonlinearly perturbed operator equations, 584

MacKichan, B. See Andreotti, A.

Mandelbaum, Richard and Moishezon, Boris. On the topological structure of simply-connected algebraic surfaces, 731

Marcus, Moshe and Mizel, Victor J. Extension theorems for nonlinear disjointly additive functionals and operators on Lebesgue spaces, with applications, 115 
Markoe, Andrew. Runge families and increasing unions of Stein spaces, 787

Marshall, Donald E. Blaschke products generate $H^{\infty}, 494$

Marx, Morris L. See Lovász, László.

Maserick, P. H. Moments of measures on convex bodies, 317

Masur, Howard. See Hubbard, John H.

Matkowsky, B. J. and Schuss, Z. On the problem of exit, 321

McCrory, Clint. Cobordism operations and singularities of maps, 281

Megiddo, Nimrod. Partial and complete cyclic orders, 274

Messer, Robert. Open three-dimensional manifolds with finitely generated fundamental groups, 89

Milman, Mario. Tensor products of function spaces, 626

Milman, V. D. See Figiel, T.

Milne, J. S. Flat homology, 118

Mizel, Victor J. See Marcus, Moshe.

Moishezon, Boris. See Mandelbaum, Richard.

Newhouse, Sheldon, Palis, Jacob and Takens, Floris. Stable arcs of diffeomorphisms, 499

Niculescu, Constantin. Banach lattices with a weak order unit, 748

Niederreiter, Harald. Statistical independence of linear congruential pseudo-random numbers, 927

Oberlin, Daniel M. Random rearrangements of Fourier coefficients, 563

Obi, W. C. See Levine, L. E.

Olin, Robert F. See Conway, John B.

de Oliveira, M. M. C. $C^{0}$-density of structurally stable vector fields, 786

Oliver, Robert. Fixed points of disk actions, 279

Palis, Jacob. See Newhouse, Sheldon

Pardon, William. Local surgery and applications to the theory of quadratic forms, 131

Parimala, S. Failure of a quadratic analogue of Serre's conjecture, 962

Pimbley, George H., Jr. A direction of bifurcation formula in the theory of the immune response, 56

Pitts, Jon T. Existence and regularity of minimal surfaces on Riemannian manifolds, 503

Plesken, Wilhelm and Pohst, Michael. On maximal finite irreducible subgroups of GL( $n, \mathrm{Z})$. I. The five and seven dimensional case. II. The six dimensional case, 757

Poénaru, V. Versal unfoldings of G-invariant functions, 86

Pohst, Michael. See Plesken, Wilhelm.

Prikry, Karel. See Jech, Thomas.

Procesi, Claudio. The invariants of $n \times n$ matrices, 891

Redheffer, Ray and Walter, Wolfgang. Existence theorems for strongly coupled systems of partial differential equations over Bernstein classes, 899

Reed, G. M. See Wage, M. L.

Reilly, Robert C. Applications of the integral of an invariant of the Hessian, 579

Riemenschneider, Oswald. Dihedral singularities: invariants, equations and infinitesimal deformations, 745

Riffelmacher, Dave. Three partial orders arising from multiplication alteration by two-cocycles, 335

Repka, Joe. Tensor products of unitary representations of $\mathrm{SL}_{2}(\mathrm{R}), 930$

Richardson, R. W. The conjugating representation of a semisimple algebraic group, 933

Roberts, John. A sufficient condition for $k$-path Hamiltonian digraphs, 63

Rodino, Luigi. See Helffer, Bernard.

Rothaus, O. S. On the nontriviality of some group extensions given by generators and relations, 284

Ruelle, D. Generalized zeta-functions for axiom A basic sets, 153

Ruittimann, Gottfried T. Stable faces of a polytope, 314

Ryff, John V. The functional equation $a f(a x)+b f(b x+a)=b f(b x)+a f(a x+b)$ extensions and almost periodic solutions, 325

Saari, Donald G. A global existence theorem for the four body problem, 743

Sacerdote, George S. A characterization of the subgroups of finitely presented groups, 609

Salinas, Norberto. Extensions of $C^{*}$-algebras and essentially n-normal operators, 143 
Schiffer, M. See Friedland, S.

Schuss, Z. See Matkowsky, B. J.

Schuur, J. See Kannan, R.

Seifert, C. J. See Diestel, J.

Shah, Jayant. Surjectivity of the period map in the case of quartic surfaces and sextic double planes, 716

Shalen, Peter B. See Jaco, William.

Shaneson, Julius L. See Cappell, Sylvain E.

Shatz, Stephen S. Degeneration and specialization in algebraic families of vector bundles, 560

Shavel, Ira. On surfaces obtained from quaternion algebras over real quadratic fields, 727

Shiffman, Bernard. Extension of holomorphic maps, 293

Singer, David. See Gluck, Herman.

Singer, David and Gluck, Herman. The existence of the nontriangulable cut loci, 599

Singer, William M. Iterated loop functors and the homology of the Steenrod algebra, 290

Slemrod, Marshall. Existence, uniqueness, stability for a simple fluid with fading memory, 581

Smith, R. T. Examples of elliptic complexes, 297

Snaith, Victor. Infinite loop maps and the complex J-homomorphism, 508

Spencer, T. See Glimm, J.

Stanojević, Časlav V. See Garrett, John W.

- See Guccione, S. J.

Stanton, Robert J. and Tomas, Peter A. Convergence of Fourier series on compact Lie groups, 61

Stein, Elliott. A smooth action on a sphere with one fixed point, 49

Stern, Ronald J. See Galewski, David E.

Stoller, Gerald S. Form of solutions to the p-adic equation $y^{\prime}=0,557$

Strömberg, Jan-Olov. Bounded mean oscillation with Orlicz norms and duality of Hardy spaces, 953

Sucheston, L. See Edgar, G. A.

Sullivan, Dennis. A new flow, 331

Sunday, Dan. The total curvature of knotted spheres, 140

Suryanarayana, M. B. Monotonicity and upper semicontinuity, 936

- See Cesari, L.

Taibleson, M. See Gandulfo, A.

Takens, Floris. See Newhouse, Sheldon.

Tanaka, Minoru. See Grove, Karsten.

Tartakoff, David S. Local Gevrey and quasi-analytic hypoellipticity for $\square_{b}, 740$

Taylor, B. A. See Bedford, Eric.

Tomas, Peter A. See Stanton, Robert J.

Travis, C. C. See Gentry, R. D.

Tromba, A. J. On the number of solutions to Plateau's problem, 66

Ulam, S. M. See Bednarek, A. R.

Wage, M. L., Fleissner, W. G. and Reed, G. M. Normality versus countable paracompactness in perfect spaces, 635

Walter, Wolfgang. See Redheffer, Ray

Ware, Buck. Infinite dimensional versions of two theorems of Carl Siegel, 613

Weinstein, Alan. The principal symbol of a distribution, 548

- See Guillemin, Victor.

Weyer, Jürgen. On domains of maximal monotone operators, 491

Wilson, Robert Lee. The roots of a simple Lie algebra are linear, 607

Wood, David H. Simple Riemann functions, 737

Wright, D. L. See Bass, H.

Wright, David. The amalgamated free product structure of $\mathrm{GL}_{2}\left(K\left[X_{1}, \ldots, X_{n}\right]\right), 724$

Wright, Marcus W. Continuity of the Kobayashi metric in deformations and the algebraic manifolds of general type, 956

Yesha, Y. See Fraenkel, A. S.

Zafran, Misha. The functions operating on certain algebras of multipliers, 939 
Zaks, Abraham. Half factorial domains, 721

Zame, William R. Existence and uniqueness of functional calculus homomorphisms, 123

Zelmanowitz, Julius. An extension of the Jacobson density theorem, 551

Ziegler, M. A language for topological structures which satisfies a Lindström-theorem, 568

Ziemer, William P. See Gariepy, Ronald.

\section{INVITED ADDRESSES}

Adler, Roy L., 472

Alperin, Jonathan L., 478

Ball, B. J. Geometric topology and shape theory: A survey of problems and results, 791

Bharucha-Reid, A. T. Fixed point theorems in probabilistic analysis, 641

Buckholtz, James D., 480

Clemens, C. H., 478

Dade, Everett C., 472

Daverman, R. D., 479

Garsia, Adriano M. Combinatorial inequalities and smoothness of functions, 157

Jaco, William, 480

Kazdan, Jerry L., 480

Kobayashi, Shoshichi. Intrinsic distances, measures and geometric function theory, 357

Kolchin, Ellis R. (Colloquium Lectures), 472

Mac Lane, Saunders. Topology and logic as a source of algebra, 1

Maskit, Bernard, 472

Mumford, David, 472

Osserman, Robert, 480

Shiffman, Bernard, 478

Silver, Jack H., 472

Stasheff, James D., 472

Stein, Elias M. (Colloquium Lectures), 472

Stoll, Wilhelm F., 472

Wallach, Nolan R. On the Selberg trace formula in the case of compact quotient, 171

Wells, R. O., Jr., 478

\section{BOOK REVIEWS}

Abhyankar, S. S. See Shatz, Stephen S.

Adams, Robert. See Ziemer, William P.

Aitchison, J. See Geisser, Seymour

Amayo, R. K. See Humphreys, J. E.

Ash, Robert B. See Taylor, S. J.

Badrikian, Albert. See Stroock, D. W.

Baker, George A. Jr. See Magnus, Arne

Balbes, Raymond. See Bennett, Mary Katherine

Barnhill, Robert E. Methods of numerical integration by Philip J. Davis and Philip Rabinowitz 538

Beck, Anatole. See Mostert, Paul S.

Bennett, Mary Katherine. Distributive lattices by Raymond Balbes and Philip Dwinger, 246

Berberian, Sterling K. Funktionalanalysis by Harro Heuser; Geometric functional analysis and its applications by Richard B. Holmes; Methods of modern mathematical physics, vol. I, Functional analysis, vol. II, Fourier analysis, self-adjointness by Michael Reed and Barry Simon, 865

Berg, Christian. See Deny, Jacques

Berkovitz, Leonard D. Deterministic and stochastic optimal control by Wendell H. Fleming and Raymond W. Rishel, 869

Birman, Joan S. See Magnus W.

Blake, Ian F. See Pless, Vera

Boas, R. P. The heat equation by D. V. Widder, 691

- Fourier series with respect to general orthogonal systems by A. M. Olevskii, 853 
Bohl, Erich. See Nashed, M. Z.

Bruneau, Michel. See Gehring, F. W. Chang, C. C. See Makkai, M.

Cheeger, Jeff. See Hermann, Robert

Chevet, Simone. See Stroock, D. W.

Comfort, W. W. Hewitt-Nachbin spaces by Maurice D. Weir, 857

Conti, R. See Sell, George R.

Copson, E. T. See Garding, Lars

Darling, D. A. Discrete-parameter martingales by J. Neveu, 836

Davis, Philip J. See Barnhill, Robert E.

Dénes, J. See Evans, Trevor

Deny, Jacques. Potential theory on locally compact abelian groups by Christian Berg and Gunnar Forst, 840

Diestel, Joseph. See Lacey, H. Elton

Dieudonné, Jean. See Hartshorne, Robin

Domb, C. See Glimm, James

Dunsmore, I. R. See Geisser, Seymour

Dwinger, Philip. See Bennett, Mary Katherine

Ebin, David G. See Hermann, Robert

Eisenstein, Gotthold. See Weil, Andre

Ellis, G. F. R. See Markus, Lawrence

Evans, Trevor. Latin squares and their applications by J. Dénes and A. D. Keedwell, 468

Ewing, John. Applications of algebraic topology by S. Lefschetz, 676

Faris, William G. See Goldstein, Jerome A.

Fink, A. M. See Sell, George R.

Fleming, Wendell H. See Berkovitz, Leonard D.

Forst, Gunnar. See Deny, Jacques

Friedman, Avner. See Varadhan, S. R. S.

Garding, Lars. Partial differential equations by E. T. Copson, 521

Gardner, Melvin B. See Taylor, S. J.

Gehring, F. W. Variation totale d'une fonction by Michel Bruneau, 532

Geisser, Seymour. Statistical prediction analysis by J. Aitchison and I. R. Dunsmore, 683

Gihman, I. I. See Stroock, D. W.

Gillman, Leonard. The Stone-Čech compactification by Russell C. Walker, 530

Glimm, James. Mathematical statistical methanics by Colin J. Thompson; Statistical mechanics and mathematical problems, Battele, Seattle edited by A. Lenard; and Phase transitions and critical phenomena edited by C. Domb and M. A. Green, 673

Goldstein, Jerome A. Self-adjoint operators by William G. Faris, 462

Govindarajulu, Z. See Wijsman, Robert A.

Gray, Brayton. See Smith, Larry

Green, M. A. See Glimm, James

Grothendieck, A. See Horváth, John

Gustafson, W. H. Maximal orders by I. Reiner, 526

Halberstam, H. H. See Montgomery, H. L.

Hale, J. K. See Sell, George R.

Hartshorne, Robin. Cours de géométrie algébrique. vols. 1 and 2 Jean Dieudonné; Basic algebraic geometry by I. R. Shafarevich, 455

Hawking, S. W. See Markus, Lawrence

Hermann, Robert. Comparison theorems in Riemannian geometry by Jeff Cheeger and David G. Ebin, 834

Heuser, Harro. See Berberian, Sterling K.

Hochstadt, Harry. See Sneddon, Ian N.

Holmes, Richard B. See Berberian, Sterling K.

Hopenwasser, Alan. Group theory and quantum mechanics by B. L. van der Waerden, 451

Horváth, John. Topological vector spaces by A. Grothendieck, 515

Humphreys, J. E. Infinite-dimensional Lie algebras by R. K. Amayo and Ian Stewart, 45

Keedwell, A. D. See Evans, Trevor 
Keisler, H. J. See Makkai, M.

Knopp, Marvin I. Elliptic modular functions by B. Schoeneberg, 218

Kuo, Hui-Hsiung. See Marcus, Michael B.

Lacey, H. Elton. Geometry of Banach spaces-Selected topics by Joseph Diestel, 681

Lang, Serge. See Langlands, R. P.

- See Roquette, Peter

Langlands, R. P. $S L_{2}$ (R) by Serge Lang, 688

Lardner, R. W. See Lowengrub, Morton

Lazard, Michel. See Lubin, Jonathan D.

Lefschetz, S. See Ewing, John

Lenard, A. See Glimm, James

Lowengrub, Morton. Mathematical theory of dislocations and fracture by R. W. Lardner, 446

Lubin, Jonathan D. Commutative formal groups by Michel Lazard, 535

Magnus, Arne. Essentials of Padé approximants by George A. Baker, Jr., 243

Magnus, W. Braids, links, and mapping class groups by Joan S. Birman, 42

Makkai, M. Model theory by C. C. Chang and H. J. Keisler, 433

Marchuk, G. I. See Wendroff, Burton

Marcus, Michael B. Gaussian measures in Banach spaces by Hui-Hsiung Kuo, 695

Markus, Lawrence. The large scale structure of space-time by S. W. Hawking and G. F. R. Ellis, 805

McShane, E. J. See Stroock, D. W,

Monna, A. F. Fourier analysis on local fields by M. H. Taibleson, 207

Montgomery, H. L. Sieve methods by H. H. Halberstam and H.-E. Richert, 846

Mosert, Paul S. Continuous flows in the plane by Anatole Beck, 693

Mullin, Ronald C. See Pless, Vera

Nashed, M. Z. Monotonie: Lösbarkeit und Numerik bei Operatorgleichungen by Erich Bohl; Iterative methods for the solution of a linear operator equation in Hilbert space-A survey by Walter Mead Patterson, 825

Neveu, J. See Darling, D. A.

Nijenhuis, Albert. See Whitehead, Earl Glen, Jr.

Nitsche, J. C. C. See Osserman, Robert

Nordgren, Eric A. Topics in operatory theory edited by C. Pearcy, 428

Olevskii, A. M. See Boas, R. P.

Orey, S. Markov chains by D. Revuz, 700

Osserman, Robert. Vorlesungen über Minimalflächen by J. C. C. Nitsche, 703

Patterson, Walter Mead. See Nashed, M. Z.

Pearcy, C. See Nordgren, Eric A.

Pless, Vera. The mathematical theory of coding by Ian F. Blake and Ronald C. Mullin, 41 Rabinowitz, Philip. See Barnhill, Robert E.

Rauch, Jeffrey. Scattering theory for the d'Alembert equation in exterior domains, by Calvin H. Wilcox, 465

Reed, Michael. See Berberian, Sterling, K.

Reiner, I. See Gustafson, W. H.

Reissig, R. See Sell, George R.

Revuz, D. See Orey, S.

Richert, H.-E. See Montgomery, H. L.

Rishel, Raymond W. See Berkovitz, Leonard D.

Ronkin, L. I. See Stoll, Wilhelm

Roquette, Peter. Elliptic functions by Serge Lang, 523

Sansone, G. See Sell, George R.

Sathaye, A. M. See Shatz, Stephen S.

Schoeneberg, B. See Knopp, Marvin I.

Schutz, J. W. See Suppes, Patrick

Seeley, R. T. Pseudo-differential operators by Michael Taylor, 249

Sell, George R. Almost periodic differential equations by A. M. Fink; Nonlinear differential equations of higher order by R. Reissig, G. Sansone and R. Conti; Functional differential equations by J. K. Hale, 198 
Shafarevich, I. R. See Hartshorne, Robin

Shatz, Stephen S. Geometric theory of algebraic space curves by S. S. Abhyankar and A. M. Sathaye, 232

Simon, Barry. See Berberian, Sterling K.

Skorohod, A. V. See Stroock, D. W.

Smith, Larry. Homotopy theory; an introduction to algebraic topology by Brayton Gray, 820 Sneddon, Ian N. The functions of mathematical physics by Harry Hochstadt, 237

Stewart, Ian. See Humphreys, J. E.

Stoll, Wilhelm. Introduction to the theory of entire functions of several variables by $\mathrm{L}$. I. Ronkin, 214

Stong, R. Algebraic topology-homotopy and homology by Robert M. Switzer, 196

Stout, William F. See Stroock, D. W.

Stroock, D. W. The theory of stochastic processes. I by I. I. Gihman and A. V. Skorohod; Stochastic calculus and stochastic models by E. J. McShane; Mesures cylindriques, espaces de Wiener, et fonctions aléatoires Gaussiennes by Albert Badrikian and Simon Chevet; Almost sure convergence by William F. Stout; Integration in Hilbert space by A. V. Skorohod, 227

Suppes, Patrick. Foundations of special relativity: Kinematic axioms for Minkowski spacetime by J. W. Schutz, 459

Switzer, Robert M. See Stong, R.

Taibleson, M. H. See Monna, A. F.

Taylor, Michael. See Seeley, R. T.

Taylor, S. J. Topics in stochastic processes by Robert B. Ash and Melvin B. Gardner, 817 Thompson, Colin J. See Glimm, James

Varadhan, S. R. S. Stochastic differential equations and applications Vols. 1 and 2 by Avner Friedman, 842

van der Waerden, B. L. See Hopenwasser, Alan

Walker, Russell C. See Gillman, Leonard

Walsh, G. R. See Young, L. C.

Weil, André. Mathematische Werke by Gotthold Eisenstein, 658

Weir, Maurice D. See Comfort, W. W.

Wendroff, Burton, Methods of numerical mathematics by G. I. Marchuk, 864

Whitehead, Earl Glen, Jr. Combinatorial algorithms by Albert Nijenhuis and Herbert S. Wilf, 870

Widder, D. V. See Boas, R. P.

Wijsman, Robert A. Sequential statistical procedures by Z. Govindarajulu, 663

Wilcox, Calvin H. See Rauch, Jeffrey

Wilf, Herbert S. See Whitehead, Earl Glen, Jr.

Young, L. C. Methods of optimization by G. R. Walsh, 540

Ziemer, William P. Sobolev spaces by Robert Adams, 668

\section{MISCELLANEOUS}

Abikoff, William. Erratum to: Augmented Teichmüller spaces, 789

Bruck, Ronald E., Jr. Corrigendum to: An iterative solution of a variational inequality for certain monotone operators in Hilbert space, 353

Council and Board of Trustees, 1975 and 1976, 354

Elections to Membership, 47, 251, 541, 872

Erratum, Volume 81, 156

Guillemin, Victor and Weinstein, Alan. Correction and addendum to: Eigenvalues associated with a closed goedesic, 966

Index to Volume 82, 968

Marcus, Moshe and Mizel, Victor J. Erratum to: Extension theorems for nonlinear disjointly additive functionals and operators on Lebesgue spaces, with applications, 353

Meetings,

The Summer meeting in Kalamazoo, 472

The October meeting in Cambridge, 478

The November meeting in Chicago, 478 
The November meeting in Blacksburg, 479

The November meeting in Los Angeles, 480

Moore, Robert Lee. See Wilder, R. L.

Reilly, Robert C. Correction to: Applications of the integral of an invariant of the Hessian, 965

Report of the Treasurer, 708

Riemenschneider, Oswald. Correction to: Dihedral singularities: Invariants, equations and infinitesimal deformations, 967

Weinstein, Alan. See Guillemin, Victor

Wilder, R. L. Robert Lee Moore, 1882-1974, 417

Zaks, Abraham. Corrigendum to: Half factorial domains, 965 